ESAIM: PROCEEDINGS, March 2012, Vol. 35, p. 1-13

Fédération Denis Poisson (Orléans-Tours) et E. Trélat (UPMC), Editors

\title{
INFORMATION ISSUES IN DIFFERENTIAL GAME THEORY*
}

\author{
Pierre Cardaliaguet ${ }^{1}$
}

\begin{abstract}
In this survey paper we present recent advances in some classes of differential game in which there is an asymmetry of information between the players. We explain that - under suitable structure conditions - these games have a value, which can be characterized in terms of (new) HamiltonJacobi equations.

Résumé. Nous présentons des résultats récents dans le domaine des jeux différentiels, pour des jeux dans lesquels les joueurs ne possèdent pas la même information sur le système. Nous expliquons que, sous certaines hypothèses de structure, ces jeux ont une valeur qui se caractérise en terme d'équations de Hamilton-Jacobi d'un type nouveau.
\end{abstract}

Differential game theory investigates conflict problems in control systems driven by several controllers. A typical differential game is the so-called pursuit-evasion game, in which one player (the pursuer) tries to capture his opponent (the evader) as fast as possible. Since the controllers have in general different aims, it is a part of game theory. In particular, it faces the same issues: how does one formalize the fact that players play simultaneously and observe each other, what is the "good" notion of solution (value, equilibria), etc ... However, because the system is in continuous time and driven by a differential equation, it has also much to do with control theory: in particular it uses the same mathematical tools, such as dynamic programming principle, Hamilton-Jacobi equations or systems, explicit computation of solutions by the method of characteristics, ... Differential game theory appeared in the early 60ies, with the contributions of Isaacs, on the one hand, and Pontryagin, on another hand, who have analyzed the first examples of such continuous-time games. There is by now a huge literature on the subject, ranging from economic applications [25], to the analysis of some partial differential equations or systems [7,9]; see also - among many other-the monographs [32,39, 40,42].

In this survey paper we concentrate on differential games in which at least one of the players knows something on the game that his opponents do not know. The general aim is to understand how this player uses this knowledge in an optimal way, and how the other players handle their lack of knowledge. Of course, stated at this level of generality, the problem is probably not even possible to formalize - let alone to solve. So we will restrict ourselves to the analysis of two player, zero sum differential games (meaning that what one wins is lost by the other). In order to understand the difficulties, let us distinguish between two different types of knowledge, that we call "information" and "observation". "Information" makes reference to the structure of the game: the game might depend on some parameters, that the informed player knows and the other player ignores: for instance, this is typically the case of a pursuer tracking a team of evaders, among which there are some decoys: the evaders know who the decoys are (the team of evaders constitute the "informed player"), but the pursuer doesn't. "Observation" refers to what the players see along the game of their opponents' behavior. In "perfect observation", they observe the other players' moves and hence know perfectly the state of

\footnotetext{
* This work was partially supported by the ANR (Agence Nationale de la Recherche) ANR-10-BLAN 0112

1 Ceremade, Université Paris-Dauphine, Place du Maréchal de Lattre de Tassigny, 75775 Paris cedex 16 - France. e-mail: cardaliaguet@ceremade.dauphine.fr
}

(C) EDP Sciences, SMAI 2012 
the system. In imperfect observation (one also says "imperfect monitoring") they observe only in a partial way their opponents and therefore do not have a complete access to the actual position of the system. For instance, in a pursuit-evasion game, the pursuer can be prevented from observing his opponent for some time because of the presence of obstacles. In both cases, the interesting point is the asymmetry of the knowledge, because players might use this asymmetry to get a better payoff: in this sense there is a strategic use of information.

If information and observation issues have been present in the problems studied by Isaacs from the beginning of the analysis on differential games, these problems are, in general, poorly understood. In most papers the authors construct a strategy for a non-fully informed controller, the other player being seen as a disturbance: see, for instance, the monograph by Baçar-Bernhard [4] and the papers by Baras-Patel [5], Baras-James [6]. In terms of game this means that one of the players does not play in an optimal way (he/she does not try to use his/her information optimally). In contrast, few works are devoted to the existence of a value for this class of games: Bernhard-Rapaport [10], on the one hand, and Petrosjan [42], on the other hand, analyze this question through some interesting classes of examples.

Games with incomplete information first appeared in repeated games theory. The simplest model is the following. Before the game starts, nature chooses at random one matrix in a finite family of matrices and tells the result to one player but not to the other. Then the players repeat infinitely often the one shot game with the chosen matrix, and, at the end, the first player gets the averaged payoff (the second player gets minus this average). This game has been studied - and solved - by Aumann-Maschler in the 60ies (see, among many other references, [3], collecting the early papers on the subject, and the monograph by Sorin [44] describing more recent advances). As far as we know, this class of games is the first one in which one can describe explicitly the strategic use of information: the main result obtained by Aumann and Maschler explains how much information the informed player has to disclose in order to optimize his payoff.

In the third section of this paper, we describe how the above model of games with incomplete information can be adapted to the framework of differential games. It turns out that, because of the continuous time structure, differential games with incomplete information present some aspects which are absent from game theory: for instance, the value function satisfies a very strange - and very degenerate-Hamilton-Jacobi (HJ) equation; the description of the first player optimal strategy involves the construction of continuous time martingale measures. The first results on differential games with incomplete information go back to [14] and [19], dealing with deterministic and stochastic differential games respectively. Since then there has been several contributions to this topic $[2,15-17,20,21,34-36,45,46]$, discussing the optimal strategies of the players, representation formula and numerical approximation.

If differential games with incomplete information are rather well understood by now, this is far from being the case for differential games with imperfect observation. The fourth section of this paper is devoted to a particular class of such games, in which one of the players is blind while the other one has a perfect observation. We show in [22] that the natural state space here is the set of probability measures on $\mathbb{R}^{N}$. As a consequence, the value function of this game can be characterized as the unique viscosity solution of some HJ equation in the Wasserstein space of probability measures.

The paper is organized as follows: in section 2 we recall the classical results on differential games with perfect information and observation: existence and characterization of the value. Section 3 is devoted to games with incomplete information: we also show, for a class of examples, how to compute the optimal strategy of the informed player. Section 4 is devoted to the game where one player has no observation at all of the system. We complete the paper with a list of open problems.

\section{Differential Games With PERFECt information}

Let us start by describing the standard method for analyzing stochastic differential games with perfect information and observation. The following technique and result go back to the seminal paper of Fleming and Souganidis [30].

We study a game in which the data are:

- a terminal time $T>0$ and an initial time $t_{0} \in[0, T]$, 
- an initial position $x_{0} \in \mathbb{R}^{N}$ and a stochastic controlled system:

$$
\left\{\begin{array}{l}
d X_{s}=b\left(s, X_{s}, u_{s}, v_{s}\right) d s+\sigma\left(s, X_{s}, u_{s}, v_{s}\right) d B_{s}, s \in[t, T] \\
X_{t_{0}}=x_{0}
\end{array}\right.
$$

where $B$ is a $d$-dimensional standard Brownian motion on a given probability space $(\Omega, \mathcal{F}, \mathbf{P}), b$ : $[0, T] \times \mathbb{R}^{N} \times U \times V \rightarrow \mathbb{R}^{N}$ and $\sigma:[0, T] \times \mathbb{R}^{N} \times U \times V \rightarrow \mathbb{R}^{N \times d}$ are smooth, bounded with bounded derivatives, maps and $U$ and $V$ being compact subsets of some finite dimensional space,

- a running and a terminal payoff $\ell:[0, T] \times \mathbb{R}^{N} \rightarrow \mathbb{R}$ and $g: \mathbb{R}^{N} \rightarrow \mathbb{R}$, also assumed to be smooth, bounded with bounded derivatives.

The players act on the system through their respective controls $\left(u_{s}\right)$ and $\left(v_{s}\right)$, which are progressively measurable processes with values in $U$ and $V$ respectively. For later use, we always denote the unique solution of (1) by $X^{t_{0}, x_{0} ; u, v}$. Under the above assumptions, it always exists.

In contrast with what happens in control theory, we now have to describe how players observe the state and their opponent's behavior. One describes this observation by the notion of strategies. Let us warn at once the reader that there is no general definition of strategies which is accepted by everyone: in fact each paper on differential game theory has its own concept of strategies, which suits its particular problem. The reason is, that it is difficult to formalize in continuous time and continuous space an observation on which one is allowed to react instantaneously.

To do so we have to introduce some notations. Let $\Omega$ be the set of continuous maps from $[0, T]$ to $\mathbb{R}^{d}$ endowed with $\mathcal{F}$, the $\sigma$-algebra generated by the coordinate process and $P$, the Wiener measure. We denote by $B$ the canonical process $B_{t}(\omega)=\omega(t)$. For fixed $t \in[0, T]$, we introduce the filtration $\mathcal{F}=\left(\mathcal{F}_{t, s}=\sigma\left\{B_{r}-B_{t}, r \in[t, s]\right\}\right.$, completed by all null sets of $P$ and denote by $\Omega_{t}$ the set of $\omega \in \Omega$ with $\omega(t)=0$.

Let $U_{t}$ (resp. $V_{t}$ ) denote the set of càdlàg maps from $[t, T]$ to $U$ (resp. $V$ ), i.e., maps which are rightcontinuous and have a left limit at each point. The sets $U_{t}, V_{t}$ and $\Omega_{t}$ are endowed with the sup-norm and the Borel $\sigma$-field generated by it. We will also need below the sets $\mathcal{U}(t)$ (resp. $\mathcal{V}(t)$ ) of adapted càdlàg processes with values in $U$ (resp. $V$ ).

Definition 1.1. Fix an initial time $t_{0} \in[0, T]$. A pure strategy for Player $I$ at time $t_{0}$ is a Borel-measurable map $\alpha: \Omega_{t_{0}} \times V_{t_{0}} \rightarrow U_{t_{0}}$ which is nonanticipative with delay, i.e., there is a partition $t_{0}<t_{1}<\cdots<t_{K}=T$ such that, for P-a.s. any $\left(\omega_{1}, \omega_{2}\right) \in \Omega^{2}$ and all $\left(v_{1}, v_{2}\right) \in V_{t_{0}}^{2}$, if $\left(\omega_{1}, v_{1}\right)=\left(\omega_{2}, v_{2}\right)$ on $\left[t_{0}, t_{i}\right]$ for some $i \in\{0, \ldots, k-1\}$, then, $\alpha\left(\omega_{1}, v_{1}\right)=\alpha\left(\omega_{2}, v_{2}\right)$ on $\left[t_{0}, t_{i+1}\right]$.

Pure strategies for Player II are defined in an analogous way. The set of pure strategies for Player I (resp. Player II) is denoted by $\mathcal{A}\left(t_{0}\right)$ (resp. $\left.\mathcal{B}\left(t_{0}\right)\right)$.

The interest of considering strategies with delay is the fact that one can associate to a pair $(\alpha, \beta)$ of strategies a unique response:

Lemma 1.2 ( [19]). Let $t_{0} \in[0, T]$ be a fixed initial time and $(\alpha, \beta) \in \mathcal{A}\left(t_{0}\right) \times \mathcal{B}\left(t_{0}\right)$ be a pair of strategies. Then there is a unique pair $(u, v) \in \mathcal{U}\left(t_{0}\right) \times \mathcal{V}\left(t_{0}\right)$ such that

$$
\alpha(v)=u \text { and } \beta(u)=v \quad \text { on }\left[t_{0}, T\right] .
$$

We set $X^{t_{0}, x_{0}, \alpha, \beta}:=X^{t_{0}, x_{0}, u, v}$ and $\left(\alpha_{s}, \beta_{s}\right):=\left(u_{s}, v_{s}\right)$, where $(u, v)$ is given by $(2)$, and define the upper and lower value functions $\mathbf{V}^{+}$and $\mathbf{V}^{-}$of the game by

$$
\begin{aligned}
& \mathbf{V}^{+}(t, x)=\inf _{\alpha \in \mathcal{A}(t)} \sup _{\beta \in \mathcal{B}(t)} \mathbf{E}\left[\int_{t}^{T} \ell\left(s, X_{s}^{t, x, \alpha, \beta}, \alpha_{s}, \beta_{s}\right) d s+g\left(X_{T}^{t, x, \alpha, \beta}\right)\right], \\
& \mathbf{V}^{-}(t, x)=\sup _{\beta \in \mathcal{B}(t)} \inf _{\alpha \in \mathcal{A}(t)} \mathbf{E}\left[\int_{t}^{T} \ell\left(s, X_{s}^{t, x, \alpha, \beta}, \alpha_{s}, \beta_{s}\right) d s+g\left(X_{T}^{t, x, \alpha, \beta}\right)\right] .
\end{aligned}
$$


Obviously, we have that $\mathbf{V}^{-}(t, x) \leq \mathbf{V}^{+}(t, x)$. The main issue in differential game theory is to prove that actually both functions coincide (one says that the game has a value function) and to characterize this value function. Another point is to describe the optimal strategies (or at least $\epsilon$-optimal strategies).

To obtain the existence of a value function, one has to assume a condition on the structure of the game: this is the so-called Isaacs' condition: for all $(t, x) \in[0, T] \times \mathbb{R}^{N}, \xi \in \mathbb{R}^{N}$, and all $A \in \mathcal{S}_{N}$ (where $\mathcal{S}_{N}$ is the set of symmetric $n \times n$ matrices), we suppose that

$$
\begin{aligned}
& H(t, x, \xi, A):= \\
& \quad \inf _{u} \sup _{v}\left\{\langle b(t, x, u, v), \xi\rangle+\frac{1}{2} \operatorname{Tr}\left(A \sigma(t, x, u, v) \sigma^{*}(t, x, u, v)\right)+\ell(t, x, u, v)\right\}= \\
& \quad \sup _{v} \inf _{u}\left\{\langle b(t, x, u, v), \xi\rangle+\frac{1}{2} \operatorname{Tr}\left(A \sigma(t, x, u, v) \sigma^{*}(t, x, u, v)\right)+\ell(t, x, u, v)\right\}
\end{aligned}
$$

This condition is trivially satisfied in the case - often encountered in practice - of separate dynamics.

Theorem 1.3 (Fleming-Souganidis [30]). Under Isaacs' condition, the game has a value: $\mathbf{V}^{+}=\mathbf{V}^{-}$in $[0, T] \times$ $\mathbb{R}^{N}$. Moreover $\mathbf{V}:=\mathbf{V}^{+}=\mathbf{V}^{-}$is the unique viscosity solution of the Hamilton-Jacobi equation:

$$
\left\{\begin{array}{l}
\mathbf{V}_{t}+H\left(t, x, D \mathbf{V}, D^{2} \mathbf{V}\right)=0 \quad \text { in }(0, T) \times \mathbb{R}^{N} \\
\mathbf{V}(T, \cdot)=g \quad \text { in } \mathbb{R}^{N}
\end{array}\right.
$$

The Hamilton-Jacobi equation is a nonlinear (often degenerate) parabolic equation: the good framework for its analysis is the notion of viscosity solutions, which has first been introduced by Crandall and Lions in the first order equations, and extended for second order equation by several authors (see for instance the famous survey by Crandall, Ishii and Lions [23] and the monographs [7,8,31]). The first application of viscosity solutions to differential games goes back to the paper by Evans and Souganidis [26]. Since then there has been a very large literature on the subject, and Theorem 1.3 has been extended to many different dynamics and payoffs: see for instance the survey paper [13] for an overview. Let us point out that, in most papers, the key assumption is always that players observe each other perfectly and have a full knowledge of the structure of the game.

To understand where this assumption plays a role, let us briefly describe the proof of Theorem 1.3: its key ingredient is the following dynamics programming principle (DPP): for any $0 \leq t_{0} \leq t_{1} \leq T$ and any $x_{0} \in \mathbb{R}^{N}$,

$$
\mathbf{V}^{+}\left(t_{0}, x_{0}\right)=\inf _{\alpha \in \mathcal{A}(t)} \sup _{\beta \in \mathcal{B}(t)} \mathbf{E}\left[\int_{t_{0}}^{t_{1}} \ell\left(s, X_{s}^{t_{0}, x_{0}, \alpha, \beta}, \alpha_{s}, \beta_{s}\right) d s+\mathbf{V}^{+}\left(t_{1}, X_{t_{1}}^{t_{0}, x_{0}, \alpha, \beta}\right)\right]
$$

while

$$
\mathbf{V}^{-}\left(t_{0}, x_{0}\right)=\sup _{\beta \in \mathcal{B}(t)} \inf _{\alpha \in \mathcal{A}(t)} \mathbf{E}\left[\int_{t_{0}}^{t_{1}} \ell\left(s, X_{s}^{t_{0}, x_{0}, \alpha, \beta}, \alpha_{s}, \beta_{s}\right) d s+\mathbf{V}^{-}\left(t_{1}, X_{t_{1}}^{t_{0}, x_{0}, \alpha, \beta}\right)\right]
$$

From this, one deduces that $\mathbf{V}^{+}$and $\mathbf{V}^{-}$satisfy the Hamilton-Jacobi equation (4), which is just an infinitesimal version of the above DPP. Since equation (4) has a unique viscosity solution, one must have $\mathbf{V}^{+}=\mathbf{V}^{-}$and the result follows. In this framework, the role of Isaacs' condition is to ensure that, despite the fact that $\mathbf{V}^{+}$ and $\mathbf{V}^{-}$satisfy a different DPP, the resulting Hamilton-Jacobi equation is the same. So the largest part of the difficulties concentrate on the one hand on the DPP, and another hand on the uniqueness of the solution of (4). For the explanation of the well-posedness of (4) we again we refer to [23]. For our purpose, it is interesting to understand what the dynamic programming means. It roughly states that, at time $t_{1}$, the players can forget all the past and play the game as if it started at that time for the position $X_{t_{1}}$. Indeed, because the players observe the state and the space, they learn nothing in the time interval $\left[t_{0}, t_{1}\right]$, so they can forget the past trajectory at time $t_{1}$ and play as if the game had freshly started at that time.

When one deals with games with incomplete information or observation, it turns out that this is no longer true, and one has to be very cautious with the dynamic programming. 


\section{Differential Games With inCOMPlete information}

Differential games with incomplete information are differential games in which at least one of the players has some private knowledge on the structure of the game: for instance, he may know precisely the realization of a random payoff or the realization of a random initial position, while the other player is only aware of the law of the payoff or of the initial position. Since we are working with two-player games, we suppose it is the first player who has some private information. Again to fix the ideas we assume that this information is on the payoff. As before we assume that the players observe each other's control perfectly. The key point is that, in this way, the non informed player can try to guess his missing information by observing the behavior of the informed player. So most of the work consists in trying to understand how the non informed player quantifies the amount of information he is learning, and, for the informed player, how much information he discloses along the game.

\subsection{Formalization and main result}

Let us now start with the formalization. As before, our game involves:

- a terminal time $T>0$ and an initial time $t_{0} \in[0, T]$,

- an initial position $x_{0} \in \mathbb{R}^{N}$ and a stochastic controlled process $\left(X_{s}\right)_{s \in\left[t_{0}, T\right]}$ driven by the stochastic differential equation (1).

The private information that the informed player (i.e., player 1) possesses is encoded as follows: we assume that there are

- a finite family of "types" $i \in\{1, \ldots, I\}$ and, for each type $i$, a running and a terminal payoff $\ell_{i}$ : $[0, T] \times \mathbb{R}^{N} \rightarrow \mathbb{R}$ and $g_{i}: \mathbb{R}^{N} \rightarrow \mathbb{R}$, which are assumed to be smooth, bounded with bounded derivatives,

- a vector $p=\left(p_{i}\right)_{i=1, \ldots, I}$ belonging to the set of probability measures $\Delta(I)$ on $\{1, \ldots, I\}$.

Heuristically the probability $p$ represents the belief of the non informed player on the type $i$. The key point is that we assume that the informed player knows also this belief. The game is played in the following way: At the initial time $t_{0}$ the type $i$ is chosen randomly accordingly to the probability $p$. The type $i$ is announced to the first player, but not to the second one. Then the players control the stochastic differential equation (1) as usual, the first player trying to minimize the payoff indexed by type $i$, while the second one seeking at maximizing it. Note however that the non informed players does not know which payoff he is actually optimizing.

In order to formalize the game, we need to modify - in fact extend - the notion of strategies introduced in Definition 1.1. Indeed, in contrast with game with perfect information, the players here can no longer play pure strategies: at least the informed player has to introduce some randomness in the game in order to hide his private information.

Definition 2.1. A random strategy is a finite distribution over pure strategies. We denote by $\mathcal{A}_{r}\left(t_{0}\right)$ (resp. $\mathcal{B}_{r}\left(t_{0}\right)$ ) the set of random strategies of player I (resp. player II) and, for $(\alpha, \beta) \in \mathcal{A}_{r}\left(t_{0}\right) \times \mathcal{B}_{r}(t)$, by $\mathbf{E}_{\alpha, \beta}$ the expectation with respect to $\mathbf{E}$ and with respect to the the probabilities associated with $\alpha$ and $\beta$.

Finally, since the first player knows the index $i$, a strategy for this player has to depend on $i$ : so it is of the form $(\alpha)_{i} \in\left(\mathcal{A}_{r}\left(t_{0}\right)\right)^{I}$. Since Player II has no private information, his strategy strategy is simply an element $\beta$ of $\mathcal{B}_{r}\left(t_{0}\right)$. The upper and lower value functions of the game are given by

$$
\begin{aligned}
\mathbf{V}^{+}\left(t_{0}, x_{0}, p\right) & =\inf _{\left(\alpha_{i}\right) \in\left(\mathcal{A}_{r}\left(t_{0}\right)\right)^{I}} \sup _{\beta \in \mathcal{B}_{r}\left(t_{0}\right)} \sum_{i=1}^{I} p_{i} \mathbf{E}_{\alpha_{i}, \beta}\left[\int_{t_{0}}^{T} \ell_{i}\left(s, X_{s}^{t_{0}, x_{0}, \alpha, \beta}, \alpha_{s}, \beta_{s}\right) d s+g\left(X_{T}^{t_{0}, x_{0}, \alpha_{i}, \beta}\right)\right], \\
\mathbf{V}^{-}(t, x, p) & =\sup _{\beta \in \mathcal{B}_{r}\left(t_{0}\right)} \inf _{\left(\alpha_{i}\right) \in\left(\mathcal{A}_{r}\left(t_{0}\right)\right)^{I}} \sum_{i=1}^{I} p_{i} \mathbf{E}_{\alpha_{i}, \beta}\left[\int_{t_{0}}^{T} \ell_{i}\left(s, X_{s}^{t_{0}, x_{0}, \alpha, \beta}, \alpha_{s}, \beta_{s}\right) d s+g\left(X_{T}^{t_{0}, x_{0}, \alpha_{i}, \beta}\right)\right] .
\end{aligned}
$$

In the above formula, the sum $\sum_{i=1}^{I} p_{i}[\cdots]$ corresponds to the expectation with respect to probability $p$. Obviously, we have that $\mathbf{V}^{-}\left(t_{0}, x_{0}, p\right) \leq \mathbf{V}^{+}\left(t_{0}, x_{0}, p\right)$ and the main part of the work consists in proving the reverse 
inequality. For this we have again to assume some Isaac' condition. It takes here the following form:

$$
\begin{aligned}
& H(t, x, \xi, A, p):= \\
& \quad \inf _{u} \sup _{v}\left\{\langle b(t, x, u, v), \xi\rangle+\frac{1}{2} \operatorname{Tr}\left(A \sigma(t, x, u, v) \sigma^{*}(t, x, u, v)\right)+\sum_{i=1}^{I} p_{i} \ell_{i}(t, x, u, v)\right\}= \\
& \quad \sup _{v} \inf _{u}\left\{\langle b(t, x, u, v), \xi\rangle+\frac{1}{2} \operatorname{Tr}\left(A \sigma(t, x, u, v) \sigma^{*}(t, x, u, v)\right)+\sum_{i=1}^{I} p_{i} \ell_{i}(t, x, u, v)\right\}
\end{aligned}
$$

for any $(t, x, \xi, A, p) \in[0, T] \times \mathbb{R}^{N} \times \mathbb{R}^{N} \times \mathcal{S}_{N} \times \Delta(I)$.

Our main result in this framework is the following:

Theorem 2.2 (Cardaliaguet-Rainer [19]). Under the above conditions, the game has a value:

$$
\mathbf{V}^{+}(t, x, p)=\mathbf{V}^{-}(t, x, p) \quad \forall(t, x, p) \in(0, T) \times \mathbb{R}^{N} \times \Delta(I)
$$

Furthermore the value $\mathbf{V}:=\mathbf{V}^{+}=\mathbf{V}^{-}$is the unique viscosity solution of the following Hamilton-Jacobi equation

$$
\left\{\begin{array}{l}
\min \left\{\mathbf{V}_{t}+H\left(t, x, D \mathbf{V}, D^{2} \mathbf{V}, p\right) ; \lambda_{\min }\left(\frac{\partial^{2} \mathbf{V}}{\partial p^{2}}\right)\right\}=0 \quad \text { in }(0, T) \times \mathbb{R}^{N} \times \Delta(I) \\
\mathbf{V}(T, \cdot, p)=\sum_{i=1}^{I} p_{i} g_{i} \quad \text { in } \mathbb{R}^{N} \times \Delta(I) .
\end{array}\right.
$$

where $\lambda_{\min }(S)$ is the minimal eigenvalue of a matrix $S$.

Let us comment upon this result. First we note that the Hamilton-Jacobi equation differs in a substantial way from the one we encountered in (4) for classical differential games: this new equation is stated in the larger state space $(0, T) \times \mathbb{R}^{N} \times \Delta(I)$. Let us also underline that the new HJ equation is much more degenerate than the original one: in particular, it is never uniformly parabolic. Its strange structure reflects the fact that we cannot expect a "standard" dynamic programming to hold. Indeed, the non informed player a priori learns something along the time from the behavior of his opponent, because the informed player cannot use his private information without revealing it, at least partially. So, if, arguing as in dynamic programming principle, the non informed player forgets about the past at an intermediated time $t_{1} \in\left(t_{0}, T\right)$, he looses some (perhaps crucial) information and plays in an non optimal way. Let us finally point out that the result says that, if there is no "classical" dynamic programming, some dynamic programming should be expected in the larger state space: although we do not know how to formalize precisely this idea yet, we give some hints of what is going on in the next subsection for a simple class of differential games.

Some words about the proof are now in order. To overcome the lack of classical dynamic programming, one first observes a key structure property of the problem: the value functions $\mathbf{V}^{+}$and $\mathbf{V}^{-}$are convex with respect to $p$. Note that this convexity is encoded in equation (5), because it implies that $\lambda_{\min }\left(\frac{\partial^{2} \mathbf{V}}{\partial p^{2}}\right) \geq 0$. Actually, the convexity of $\mathbf{V}^{-}$in $p$ can be easily derived from its definition. It is not so simple to check (before knowing that the game has a value) that $\mathbf{V}^{+}$is convex in $p$ : one has to use the so-called splitting method, introduced for repeated games with incomplete information by Aumann-Maschler [3].

Because of this convexity property, one is lead to introduce, as in De Meyer for repeated games [24], the dual Fenchel conjugate of $\mathbf{V}^{-}$with respect to $p$ :

$$
\mathbf{V}^{-*}(t, x, \hat{p})=\sup _{p \in \Delta(I)}\langle\hat{p}, p\rangle-\mathbf{V}^{-}(t, x, p) \quad \forall(t, x, \hat{p}) \in[0, T] \times \mathbb{R}^{N} \times \mathbb{R}^{I}
$$

It turns out that $\mathbf{V}^{-*}$ is the (upper-)value function of an unusual differential game: namely

$$
\mathbf{V}^{-*}(t, x, \hat{p})=\inf _{\beta \in \mathcal{B}_{r}(t)} \sup _{\alpha \in \mathcal{A}_{r}(t)} \max _{i \in\{1, \ldots, I\}}\left\{\hat{p}_{i}-\mathbf{E}_{\alpha \beta}\left[\int_{t}^{T} \ell_{i}\left(s, X_{s}^{t, x, \alpha, \beta}, \alpha_{s}, \beta_{s}\right)+g_{i}\left(X_{T}^{t, x, \alpha, \beta}\right)\right]\right\}
$$


This problem is really awkward because the criterium is not an expectation, but the maximum of expectations. Using this formulation, one can then show that $\mathbf{V}^{-*}$ satisfies the subdynamic programming inequality

$$
\mathbf{V}^{-*}\left(t_{0}, x_{0}, \hat{p}\right) \leq \inf _{\beta \in \mathcal{B}\left(t_{0}\right)} \sup _{\alpha \in \mathcal{A}\left(t_{0}\right)} \mathbf{E}\left[\mathbf{V}^{-*}\left(t_{1}, X_{t_{1}}^{t_{0}, x_{0}, \alpha, \beta}, \hat{p}\left(t_{1}\right)\right)\right]
$$

where

$$
\hat{p}\left(t_{1}\right)_{i}=\hat{p}_{i}-\int_{t_{0}}^{t_{1}} \ell_{i}\left(s, X_{s}^{t_{0}, x_{0}, \alpha, \beta}, \alpha_{s}, \beta_{s}\right) d s \quad \text { for } i=1, \ldots, I .
$$

From this one derives that $\mathbf{V}^{-}$is a viscosity subsolution of (5). One also proves, in a simpler way, that $\mathbf{V}^{+}$ satisfies a subdynamic programming inequality and is a subsolution of (5). One then concludes by proving a comparison property of equation (5), which says - as usual - that subsolutions are below supersolutions. Hence $\mathbf{V}^{+} \leq \mathbf{V}^{-}$, which was the missing inequality.

In terms of PDE, the comparison theorem for (5) is quite intricate because of the strong degeneracy of the equation. Several equivalent definition can be given, some being stated for the convex conjugate instead of the function itself. The formulation presented here follows [16].

\subsection{An example of continuous-time game without dynamics}

Up to now, we have characterized the value function. This characterization however does not give any clue on what the players have to play. In order to illustrate the previous results and understand the players' optimal strategies, we now consider a simple class of games: a two-player zero-sum continuous time game without dynamics, in which the first player has a private information on the random running payoff. The description of the game involves

(i) an initial time $t_{0} \geq 0$ and a terminal time $T>t_{0}$,

(ii) a finite number $I$ integral payoffs (where $I \geq 2$ ) $\ell_{i}:[0, T] \times U \times V \rightarrow \mathbb{R}$ for $i=1, \ldots I$, where $U$ and $V$ are compact subsets of some finite dimensional spaces,

(iii) a probability $p=\left(p_{i}\right)_{i=1, \ldots, I}$ belonging to the set $\Delta(I)$ of probabilities on $\{1, \ldots, I\}$.

As before the game is played in two steps: at time $t_{0}$, the index $i$ is chosen at random among $\{1, \ldots, I\}$ according to probability $p$; the choice of $i$ is communicated to Player 1 only. Then the players choose their respective controls in order, for Player 1, to minimize the integral payoff $\int_{t_{0}}^{T} \ell_{i}(s, u(s), v(s)) d s$, and for Player 2 to maximize it. We again assume that both players observe their opponent's control. Note however that Player 2 does not know which payoff he/she is actually maximizing.

The existence and the characterization of a value for this game is a particular case of Theorem 2.2: if Isaacs' condition holds:

$$
H(t, p)=\inf _{u \in U} \sup _{v \in V} \sum_{i=1}^{I} p_{i} \ell_{i}(t, u, v)=\sup _{v \in V} \inf _{u \in U} \sum_{i=1}^{I} p_{i} \ell_{i}(t, u, v) \quad \forall(t, p) \in[0, T] \times \Delta(I)
$$

the game has a value $\mathbf{V}=\mathbf{V}\left(t_{0}, p\right)$ given by

$$
\begin{array}{r}
\mathbf{V}\left(t_{0}, p\right)=\inf _{\left(\alpha_{i}\right) \in\left(\mathcal{A}_{r}\left(t_{0}\right)\right)^{I}} \sup _{\beta \in \mathcal{B}_{r}\left(t_{0}\right)} \sum_{i=1}^{I} p_{i} \mathbf{E}_{\alpha_{i} \beta}\left[\int_{t_{0}}^{T} \ell_{i}\left(s, \alpha_{i}(s), \beta(s)\right) d s\right] \\
=\sup _{\beta \in \mathcal{B}_{r}\left(t_{0}\right)} \inf _{\left(\alpha_{i}\right) \in\left(\mathcal{A}_{r}\left(t_{0}\right)\right)^{I}} \sum_{i=1}^{I} p_{i} \mathbf{E}_{\alpha_{i} \beta}\left[\int_{t_{0}}^{T} \ell_{i}\left(s, \alpha_{i}(s), \beta(s)\right) d s\right],
\end{array}
$$


for any $\left(t_{0}, p\right) \in[0, T] \times \Delta(I)$. This value can be characterized as the unique viscosity solution of the HJ equation

$$
\left\{\begin{array}{l}
\min \left\{\mathbf{V}_{t}+H(t, p), \lambda_{\min }\left(\frac{\partial^{2} \mathbf{V}}{\partial p^{2}}\right)\right\}=0 \quad \text { in }(0, T) \times \Delta(I) \\
\mathbf{V}(T, \cdot)=0 \quad \text { in } \Delta(I)
\end{array}\right.
$$

Our first aim is to compute explicitly $\mathbf{V}$ under some conditions.

Example 2.3. Assume that $H$ does not depend on $t$. Then $\mathbf{V}(t, p)=(T-t) \operatorname{Vex} H(p)$, where $\operatorname{Vex} H(p)$ denote the convex hull of $H(\cdot)$.

Indeed the map $w(t, p)=(T-t) \operatorname{Vex} H(p)$ is convex in $p$ and satisfies $w(T, p)=0$. Moreover, since $w_{t}=$ - Vex $H(p)$, either $\operatorname{Vex} H(p)=H(p)$, and then $w_{t}+H(p)=0$, or $\operatorname{Vex} H(p)<H(p)$, and then $\operatorname{Vex} H(\cdot)$ must be flat near $p$, so that $\lambda_{\min }\left(\frac{\partial^{2} w}{\partial p^{2}}\right)=0$ (in the viscosity sense). In both cases equality $\min \left\{\mathbf{V}_{t}+H(t, p), \lambda_{\min }\left(\frac{\partial^{2} \mathbf{V}}{\partial p^{2}}\right)\right\}=$ 0 holds. By characterization, this implies that $\mathbf{V}=w$.

Example 2.4. Assume that $I=2$ and that there exists $h_{1}, h_{2}:[0, T] \rightarrow[0,1]$ continuous, $h_{1} \leq h_{2}, h_{1}$ decreasing and $h_{2}$ increasing, such that

$$
\operatorname{Vex} H(t, p)=H(t, p) \Leftrightarrow p \in\left[0, h_{1}(t)\right] \cup\left[h_{2}(t), 1\right]
$$

and

$$
\frac{\partial^{2} H}{\partial p^{2}}(t, p)>0 \quad \forall(t, p) \text { with } p \in\left[0, h_{1}(t)\right) \cup\left(h_{2}(t), 1\right] .
$$

Then the reader can check, as in Example 2.3, that

$$
\mathbf{V}(t, p)=\int_{t}^{T} \operatorname{Vex} H(s, p) d s \quad \forall(t, p) \in[0, T] \times \Delta(I) .
$$

In order to describe the strategy of the informed player, we need to provide a further characterization of the value. For this let us introduce some notations: Let $\mathbf{D}\left(t_{0}\right)$ be the set of càdlàg functions from $\mathbb{R}$ to $\Delta(I)$ which are constant on $\left(-\infty, t_{0}\right)$ and on $[T,+\infty)$, let $t \mapsto \mathbf{p}(t)$ be the coordinate mapping on $\mathbf{D}\left(t_{0}\right)$ and let $\mathcal{G}=\left(\mathcal{G}_{t}\right)$ be the filtration generated by $t \mapsto \mathbf{p}(t)$.

Given $p_{0} \in \Delta(I)$, we denote by $\mathbf{M}\left(t_{0}, p_{0}\right)$ the set of probability measures $\mathbf{P}$ on $\mathbf{D}\left(t_{0}\right)$ such that, under $\mathbf{P}$, $(\mathbf{p}(t), t \in[0, T])$ is a martingale and satisfies :

$$
\text { for } t<t_{0}, \mathbf{p}(t)=p_{0} \quad \text { and, for } t \geq T, \mathbf{p}(t) \in\left\{e_{i}, i=1, \ldots, I\right\} \quad \mathbf{P} \text {-a.s. . }
$$

Finally for any measure $\mathbf{P}$ on $\mathbf{D}\left(t_{0}\right)$, we denote by $\mathbf{E}_{\mathbf{P}}[\ldots]$ the expectation with respect to $\mathbf{P}$. Heuristically, the probability measure $\mathbf{P}$ represents the amount of information the informed players discloses along the time. This interpretation is justified by the following result:

Theorem 2.5 (Cardaliaguet-Rainer [20]).

$$
\mathbf{V}\left(t_{0}, p_{0}\right)=\min _{\mathbf{P} \in \mathbf{M}\left(t_{0}, p_{0}\right)} \mathbf{E}_{\mathbf{P}}\left[\int_{t_{0}}^{T} H(s, \mathbf{p}(s)) d s\right] \quad \forall\left(t_{0}, p_{0}\right) \in[0, T] \times \Delta(I) .
$$

Note that, with this formulation, the differential game problem becomes a control problem in which the family of controls are martingale measures. One of the main interests of Theorem 2.5 is that it allows to describe the optimal strategy of the informed player: Let $\left(t_{0}, p_{0}\right) \in[0, T) \times \Delta(I)$ be fixed, $\overline{\mathbf{P}}$ be optimal in the problem (11). Let us set $E_{i}=\left\{\mathbf{p}(T)=e_{i}\right\}$ and define the probability measure $\overline{\mathbf{P}}_{i}$ by: $\forall A \in \mathcal{G}, \overline{\mathbf{P}}_{i}(A):=\overline{\mathbf{P}}\left[A \mid E_{i}\right]=\frac{\overline{\mathbf{P}}\left(A \cap E_{i}\right)}{p_{i}}$, 
if $p_{i}>0$, and $\overline{\mathbf{P}}_{i}(A)=P(A)$ for an arbitrary probability measure $P \in \mathbf{M}\left(t_{0}, p_{0}\right)$ if $p_{i}=0$.

We also set

$$
\overline{\mathbf{u}}(t)=u^{*}(t, \mathbf{p}(t)) \quad \forall t \in \mathbb{R},
$$

where $u^{*}=u^{*}(t, p)$ is a Borel measurable selection of $\operatorname{argmin}_{u \in U}\left(\max _{v \in V} \sum_{i=1}^{I} p_{i} \ell_{i}(t, u, v)\right)$ and where we denote by $\overline{\mathbf{u}}_{i}$ the random control $\overline{\mathbf{u}}_{i}=\left(\left(\mathbf{D}\left(t_{0}\right), \mathcal{G}, \overline{\mathbf{P}}_{i}\right), \overline{\mathbf{u}}\right) \in \mathcal{U}_{r}\left(t_{0}\right)$.

Theorem 2.6 ( $[20])$. The strategy consisting in playing the random control $\left(\overline{\mathbf{u}}_{i}\right)_{i=1, \ldots, I} \in\left(\mathcal{U}_{r}\left(t_{0}\right)\right)^{I}$ is optimal for $\mathbf{V}\left(t_{0}, p_{0}\right)$. Namely

$$
\mathbf{V}\left(t_{0}, p_{0}\right)=\sup _{\beta \in \mathcal{B}_{r}\left(t_{0}\right)} \sum_{i=1}^{I} p_{i} \mathbf{E}_{\overline{\mathbf{u}}_{i}}\left[\int_{t_{0}}^{T} \ell_{i}\left(s, \overline{\mathbf{u}}_{i}(s), \beta\left(\overline{\mathbf{u}}_{i}\right)(s)\right) d s\right] .
$$

Since the computation of the optimal strategy of the informed player can be reduced to the computation of the optimal martingale measure in (11), it is interesting to have a full characterization of such measure. In [20] we give some necessary and some sufficient conditions for a measure to be optimal. In the case of Example 2.4 it is possible to identify explicitly this optimal martingale measure: it mainly lives on the graph of the maps $h_{1}$ and $h_{2}$.

Proposition 2.7. Under the assumptions of Example 2.4, there is a unique optimal martingale measure $\overline{\mathbf{P}}$. Under this martingale measure, the process $\mathbf{p}$ is purely discontinuous and satisfies:

$$
\mathbf{p}\left(t^{-}\right)=p_{0} \quad \forall t \in\left[t_{0}, t^{*}\right] \overline{\mathbf{P}}-\text { a.s., where } t^{*}=\inf \left\{t \geq t_{0} \mid p_{0} \in\left[h_{1}(t), h_{2}(t)\right]\right\}
$$

and

$$
\mathbf{p}(t) \in\left\{h_{1}(t), h_{2}(t)\right\} \quad \forall t \in\left[t^{*}, T\right) \overline{\mathbf{P}}-\text { a.s. } .
$$

We now turn to the optimal strategy of the non informed player. This strategy has been studied by Souquière [46]. Let us first remark that we can work with the dual problem (recall (6), in which we set for simplicity $\left.\mathbf{V}^{*}:=\mathbf{V}^{-*}\right)$ :

Lemma 2.8. Let $\left(t_{0}, x_{0}, p_{0}\right) \in[0, T) \times \mathbb{R}^{N} \times \Delta(I)$ and $\hat{p}$ be an element of the subdifferential of the convex map $p \rightarrow \mathbf{V}\left(t_{0}, x_{0}, p\right)$ at $p_{0}$. If a strategy $\bar{\beta} \in \mathcal{B}_{r}(t)$ is optimal for $\mathbf{V}^{*}\left(t_{0}, x_{0}, \hat{p}\right)$, then it is optimal for $\mathbf{V}\left(t_{0}, x_{0}, p_{0}\right)$.

So we only need to work for the dual game (7). We have seen in section 2.1 that the value function of the dual game satisfies the subdynamic principle (8). It turns out that, in the particular case of the value function given by (10), we actually have a dynamic programming:

Lemma 2.9. The dual value function $\mathbf{V}^{*}$ is the unique viscosity solution of the following HJ equation:

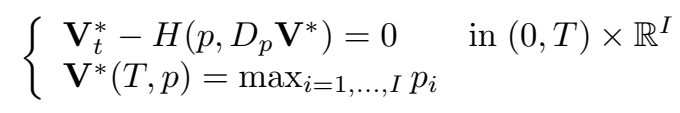

This means that $\mathbf{V}^{*}$ is just the value function of a standard differential game: one can then use standard tools for computing the optimal strategies. In particular, as explained in section 1, these strategies need not be random.

Actually, one can be more precise: indeed Hamilton-Jacobi equation (13) has a specific structure, it is positively homogeneous with respect to the gradient variable. Hence equation (13) is a level-set equation (also called geometric equation). This means that a level set of the solution only depends on the corresponding level-set for the terminal data. So an optimal strategy for the non informed player just consists in remains in the given level-set. This idea is very close to the notion of "approachability" developed for repeated games by Blackwell (see for instance [44]) but also to the notion of stable bridge developed in differential game theory by Krasovskii-Subbotin [40]: see Souquière [46] for more precise statements. 


\subsection{Further results}

The results of this section have been extended in several directions. First it holds even if both players have a private information and if this private information is on the running and the terminal payoff and on the initial position. The infinite horizon problem has also been considered in As Soulaimani [2]. Some representation formula - of Hopf-Lax type - are given in Cardaliaguet [15] and Souquière [45], while the construction of $\epsilon$-optimal strategies and approximation of the value function are carried out in Cardaliaguet [17], Souquière [45] and Grün [35].

The simple example described in subsection 2.2 has been extended in two directions. First it is natural to study what happens when the family of types $\{1, \ldots, I\}$ is no longer finite [21]. In this situation, the HJ equation is stated in the space of measures and the characterization Theorem 2.5 becomes a minimization problem over the class of martingale measures. Second, another (and more striking) extension has been found by Grün [34] for stochastic differential games in which the volatility term $\sigma$ is non degenerate and does not depend upon the control variables. In this case, one can use Girsanov transform and backward stochastic differential equation (BSDE) to reformulate the game, in the same spirit as in Hamadène, Lepeltier, Peng [37]. The value function can again be rewritten as a minimization problem over a class of martingale measures, but the criteria now depends on the solution of a BSDE. One can derive from this approach a new numerical method [35] as well as a surprising regularity result for the value function [36].

\section{A DifFERENTIAL GAME With INCOMPlete OBSERVATION}

If differential games with incomplete information are rather well understood by now, this is not at all the case for games with incomplete observation: in this case one of the player does not observe his opponent perfectly, and the main issue is to understand what is the proper "extended state space". We now describe a result given in Cardaliaguet-Souquière [22] in which we assume that one of the players (player 2) is blind: he does not observe his opponent at all. The first player, on the contrary, observes his opponent and the state of the problem. Again we assume that the non informed player has a probabilistic model of the distribution of the state at the initial time, and that the informed player knows this distribution. For simplicity we work with deterministic dynamics.

Let us now start with the formalization. As before, our game involves:

- a terminal time $T>0$ and an initial time $t_{0} \in[0, T]$,

- a (deterministic) differential equation

$$
\left\{\begin{array}{l}
d X_{s}=b\left(s, X_{s}, u_{s}, v_{s}\right) d s, s \in[t, T] \\
X_{t_{0}}^{t_{0}, x_{0} ; u, v}=x_{0}
\end{array}\right.
$$

- a terminal payoff $g: \mathbb{R}^{N} \rightarrow \mathbb{R}$.

As before, for a given initial position $x_{0} \in \mathbb{R}^{N}$, we denote by $X^{t_{0}, x_{0} ; u, v}$ the unique solution to (14). The private information that the informed player (i.e., player 1) possesses is encoded as follows: we assume that, at initial time $t_{0}$, the initial position $x_{0}$ is chosen randomly according to a probability measure $\mu_{0}$ on $\mathbb{R}^{N}$ known by both players. The position $x_{0}$ is known by the first player, but not by the second one. We assume that the first player is minimizing the terminal payoff $g\left(X_{T}\right)$ while the first player is optimizing it.

As before we have to define the notion of strategies. Since the second player does not observe anything, a strategy for this player is simply a probability measure $Q$ on the set $\mathcal{V}\left(t_{0}\right)$ of open loop controls. We denote by $\Delta\left(\mathcal{V}\left(t_{0}\right)\right)$ is the set of strategies of Player 2. For the first player, a strategy with delay $\tau$ is a measurable map $(x, v) \rightarrow P_{x}^{v}$ from $\mathbb{R}^{N} \times \mathcal{V}\left(t_{0}\right)$ into the set $\Delta\left(\mathcal{U}\left(t_{0}\right)\right)$ of probability measures on $\mathcal{U}\left(t_{0}\right)$ with the nonanticipativity property: if $v_{1} \equiv v_{2}$ a.e. on $\left[t_{0}, t\right]$, then $P_{x}^{v_{1}} \equiv P_{x}^{v_{2}}$ on $\left[t_{0}, t+\tau\right]$ for any $x \in \mathbb{R}^{N}$. We denote by $\Delta\left(\mathcal{A}_{x}^{\tau}\left(t_{0}\right)\right)$ the set of strategies of Player 1 with delay $\tau$ and we set

$$
\Delta\left(\mathcal{A}_{x}\left(t_{0}\right)\right)=\bigcup_{\tau>0} \Delta\left(\mathcal{A}_{x}^{\tau}\left(t_{0}\right)\right) .
$$


Given two strategies $(P, Q) \in \Delta\left(\mathcal{A}_{x}\left(t_{0}\right)\right) \times \Delta\left(\mathcal{V}\left(t_{0}\right)\right)$, we set

$$
\mathfrak{J}\left(t_{0}, \mu_{0}, P, Q\right)=\int_{\mathbb{R}^{N} \times \mathcal{U}\left(t_{0}\right) \times \mathcal{V}\left(t_{0}\right)} g\left(X_{T}^{t_{0}, x, u, v}\right) d P_{x}^{v}(u) d Q(v) d \mu_{0}(x)
$$

Then the lower value and the upper value of the game are given by:

$$
\begin{aligned}
\mathbf{V}^{-}\left(t_{0}, \mu_{0}\right) & =\lim _{\tau \rightarrow 0^{+}} \sup _{Q \in \Delta\left(\mathcal{V}\left(t_{0}\right)\right)} \inf _{P \in \Delta\left(\mathcal{A}_{x}^{\tau}\left(t_{0}\right)\right)} \mathfrak{J}\left(t_{0}, \mu_{0}, P, Q\right), \\
\mathbf{V}^{+}\left(t_{0}, \mu_{0}\right) & =\lim _{\tau \rightarrow 0} \inf _{P \in \Delta\left(\mathcal{A}_{x}^{\tau}\left(t_{0}\right)\right)} \sup _{Q \in \Delta\left(\mathcal{V}\left(t_{0}\right)\right)} \mathfrak{J}\left(t_{0}, \mu_{0}, P, Q\right) \\
& =\inf _{P \in \Delta\left(\mathcal{A}_{x}\left(t_{0}\right)\right)} \sup _{v \in \mathcal{V}\left(t_{0}\right)} \mathfrak{J}\left(t_{0}, \mu_{0}, P, v\right)
\end{aligned}
$$

Throughout this section we assume that the measure $\mu_{0}$ is a Borel probability measure with finite second order moment: $\int_{\mathbb{R}^{N}}|x|^{2} d \mu_{0}(x)<\infty$. We denote by $\mathcal{W}$ the set of such measures. This allows to work with Wasserstein distance on $\mathcal{W}$ :

$$
\mathbf{d}^{2}(\mu, \nu)=\min _{\pi \in \Pi(\mu, \nu)} \int_{\mathbb{R}^{2 N}}|x-y|^{2} d \pi(x, y) \quad \forall \mu, \nu \in \mathcal{W}
$$

where $\Pi(\mu, \nu)$ is the set of probability measures on $\mathbb{R}^{N} \times \mathbb{R}^{N}$ with marginals $\mu$ and $\nu$.

Theorem 3.1 (Cardaliaguet-Souquière $[22]$ ). For all $(t, \mu) \in[0, T] \times \mathcal{W}$,

$$
\mathbf{V}^{+}(t, \mu)=\mathbf{V}^{-}(t, \mu)
$$

Moreover $\mathbf{V}:=\mathbf{V}^{+}=\mathbf{V}^{-}$is the unique viscosity solution of the HJ equation

$$
\left\{\begin{array}{l}
\mathbf{V}_{t}+\mathcal{H}\left(\mu, D_{\mu} \mathbf{V}\right)=0 \quad \text { in }(0, T) \times \mathcal{W} \\
\mathbf{V}(T, \mu)=\int_{\mathbb{R}^{N}} g(x) d \mu(x) \quad \text { in } \mathcal{W}
\end{array}\right.
$$

where, for any $\mu \in \mathcal{W}$ and $\mathbf{p} \in L_{\mu}^{2}\left(\mathbb{R}^{N}, \mathbb{R}^{N}\right)$,

$$
\mathcal{H}(\mu, \mathbf{p})=\sup _{v \in V} \int_{\mathbb{R}^{N}} \inf _{u \in U}\langle f(x, u, v), \mathbf{p}(x)\rangle d \mu(x) .
$$

As explained in for instance in [1] one can naturally endow the Wasserstein space $\mathcal{W}$ with a differential structure and, therefore, write HJ equations on this space. Several definitions of viscosity solution have been proposed in this framework: see for instance [27-29,33,41]. The definition and the comparison principle used here are borrowed from Cardaliaguet-Quincampoix [18].

What is surprising in the above result is the fact that there is a HJ equation: indeed the second player observes nothing, so, apparently, he cannot update his knowledge on the state. This is however not true for the lower value: indeed, by definition of $\mathbf{V}^{-}$, the second player knows his opponent's strategy and therefore can update at each time the distribution of the position of the state. This gives the dynamic programming in the space of measures $\mathcal{W}$ and the resulting HJ equation. In fact, as in the usual cases, one can describe the informed player's strategy by using the HJ equation. The difficulty arises with the upper value $\mathbf{V}^{+}$, for which we do not know how to prove that it satisfies a dynamic programming before showing that the game has a value. Therefore we cannot rely on the PDE techniques for proving the existence of this value and have to go back to a min-max Theorem. Let us finally point out that, if we can describe, using the HJ equation, the optimal strategy of the first player, we have no clue about the non informed player's strategy. 


\section{OPEN PROBLEMS}

To complete the paper we give a list of open problems on differential games with informations issues. Some of them (the first ones) are clearly tractable. The last ones are probably more intricate.

- (Direct dynamic programming approach) The proofs for the existence of a value given in [14] and [20] rely on a (sub)dynamic programming principle for the value of the dual game (i.e., $\mathbf{V}^{-*}$ ). It would be very interesting to write a dynamic programming (in)equalities for the value function itself: this dynamic programming should involve an update of the information by the non informed player.

- (Optimal strategies for the non informed player) We have seen that it is crucial for the informed player to hide his information by using random strategies. It is less clear that the non informed player should play a random strategy because he has nothing to hide in principle. For instance, for the simple game given in subsection 2.2, we have seen that the optimal strategy of the non informed player needs no randomness. We do not know if this is the case in general.

- (General informations) So far, we have assumed that the information set $I$ is disclosed to the informed player at the initial time. It would be interesting to investigate what happens in the more natural situation where the information is disclosed all along the game. For instance, the informed player (and not the other player) could observe a auxiliary random process on which the payoff depends.

- (Non zero sum differential games) There are several approaches to non zero differential games with perfect information (by PDE techniques [9,11], by BSDEs methods [38], by a folk Theorem [12],...). The case of incomplete information is completely open, although the existence of equilibria is known for repeated games with lack of information (Simon-Spiez-Toruńczyk [43]).

- (Imperfect monitoring) Beside the case of games with a blind player described above, basically nothing is known on differential games with imperfect observation.

\section{REFERENCES}

[1] L. Ambrosio, N. Gigli, G. Savaré, Gradient flows in metric spaces and in the space of probabiliy measures, Lectures Math. ETH Zürich, Birkhäuser Verlag, Basel, 2005.

[2] As Soulaimani, S. Approchabilité, Viabilité et Jeux Différentiels en Information Incomplète, Thèse de l'Université de Brest, 2008.

[3] Aumann, R. J.; Maschler, M. B. Repeated games with incomplete information. With the collaboration of Richard E. Stearns. MIT Press, Cambridge, MA, 1995.

[4] Baçar, T., Bernhard, P. $H^{\infty}$-optimal control and related minimax design problems. A dynamic game approach. Second edition. Systems \& Control: Foundations \& Applications. Birkhäuser Boston, Inc., Boston, MA, 1995.

[5] Baras, J. S.; Patel, N. S. Robust control of set-valued discrete-time dynamical systems, IEEE Trans. Automat. Control, 43, pp. 61-75, 1998.

[6] Baras, J. S., James, M. R. Partially observed differential games, infinite-dimensional Hamilton-Jacobi-Isaacs equations, and nonlinear $H_{\infty}$ control, SIAM J. Control Optim., 34, pp. 1342-1364, 1996.

[7] Bardi M. , Capuzzo Dolcetta I. Optimal control and viscosity solutions of Hamilton-Jacobi-Bellman equations. Birkhäuser, 1996.

[8] Barles G. Solutions de viscosité des équations de Hamilton-Jacobi. Springer-Verlag, Berlin, 1994.

[9] Bensoussan, A., Frehse, J. Regularity results for nonlinear elliptic systems and applications. Applied Mathematical Sciences, 151. Springer-Verlag, Berlin, 2002.

[10] Bernhard, P., Rapaport, A. Min Max Certainty equivalence principle and Differential Games, International Journal of Robust and Nonlinear Control 96, no. 8, pp. 825-842, 1996.

[11] Bressan, A., Shen W. Small BV solutions of hyperbolic non-cooperative differential games, SIAM J. Control Optim. 43, 104-215, 2004.

[12] Buckdahn, R., Cardaliaguet P., Rainer C. Nash equilibrium payoffs for nonzero-sum stochastic differential games, SIAM J. Control Optim. 43, no. 2, 624-642, 2004.

[13] Buckdahn, R., Cardaliaguet P., Quincampoix M. Some Recent Aspects of Differential Game Theory. Dyn Games Appl, 1: 74-114, 2011.

[14] Cardaliaguet P. Differential games with asymmetric information. SIAM J. Control Optim. 46, no. 3, 816-838, 2007.

[15] Cardaliaguet P. Representation formulas for differential games with asymmetric information. J. Optim. Theory Appl. 138, no. 1, 1-16, 2008. 
[16] Cardaliaguet P. A double obstacle problem arising in differential game theory. J. Math. Anal. Appl. 360, no. 1, 95-107, 2009.

[17] Cardaliaguet P., Numerical Approximation and Optimal Strategies for Differential Games with Lack of Information on One Side. Annals of ISDG, 10, 159-176, 2009.

[18] Cardaliaguet P., Quincampoix M. Deterministic differential games under probability knowledge of initial condition. Int. Game Theory Rev. 10, no. 1, 1-16, 2008.

[19] Cardaliaguet P., Rainer C. Stochastic differential games with asymmetric information. Appl. Math. Optim. 59: 1-36, 2009.

[20] Cardaliaguet P.; Rainer C. On a continuous time game with incomplete information. Math. Oper. Res. 34, no. 4, 769-794, 2009.

[21] Cardaliaguet P.; Rainer C. Games with incomplete information in continuous time and for continuous types. Pre-print, 2012.

[22] Cardaliaguet P.; Souquière A. Differential games with a blind player. Pre-print, 2010.

[23] Crandall M.G., Ishii H., Lions P.-L. User's guide to viscosity solutions of second order Partial Differential Equations. Bull. Amer. Soc., 27, pp. 1-67, 1992.

[24] De Meyer, B. Repeated games, duality and the central limit theorem. Math. Oper. Res. 21, no. 1, 237-251, 1996.

[25] Dockner, E., Jorgensen, S., Van Long, N., Sorger, G. Differential games in economics and management science. Cambridge University Press, 2000.

[26] Evans, L.C., Souganidis, P.E. Differential games and representation formulas for solutions of Hamilton-Jacobi Equations. Indiana Univ. Math. J., 282, pp. 487-502, 1984.

[27] Feng, J. and Kurtz T. G. Large deviations for stochastic processes, volume 131 of Mathematical Surveys and Monographs. American Mathematical Society, 2006.

[28] Feng J., Katsoulakis M. A Comparison Principle for Hamilton-Jacobi Equations Related to Controlled Gradient Flows in Infinite Dimensions, Arch. Rational Mech. Anal. 192, 275-310, 2009.

[29] Feng J., Swiech A. Optimal control for a mixed flow of Hamiltonian and gradient type in space of probability measures. Trans. Amer. Math. Soc.

[30] Fleming, W.H.; Souganidis, P.E. On the existence of value functions of two-player, zero-sum stochastic differential games. Indiana Univ. Math. J. 38, No.2, 293-314, 1989.

[31] Fleming W.H., Soner H.M. Controlled Markov processes and viscosity solution. Springer-Verlag, New-York, 1993.

[32] Friedman A. Differential Games. Wiley, New York, 1971.

[33] Gangbo W., Nguyen T., Adrian T. A. Hamilton-Jacobi in the Wasserstein space, Methods and Applications in Analysis, Vol. 15, No. 2, pp. 155-184, 2008.

[34] C. Grün, A BSDE approach to stochastic differential games with incomplete information, To appear in Stochastic Processes an Their Applications.

[35] C. Grün, Probabilistic numerical scheme for an obstacle problem arising in game theory. Preprint, 2011.

[36] C. Grün, A regularity result for a fully non-linear PDE related to game theory. Preprint, 2011.

[37] Hamadène, S., Lepeltier, J.-P., Peng, S. BSDEs with continuous coefficients and stochastic differential games. El Karoui, Nicole (ed.) et al., Backward stochastic differential equations. Harlow: Longman. Pitman Res. Notes Math. Ser. 364, 115-128, 1997.

[38] Hamadène, S. Nonzero sum linear-quadratic stochastic differential games and backward-forward equations. Stochastic Anal. Appl. 17, No.1, 117-130, 1999.

[39] Isaacs R. Differential Games. Wiley, New York, 1965.

[40] Krasovskii N.N., Subbotin A.I. Game-Theorical Control Problems. Springer-Verlag, New-York, 1988.

[41] Lions, P.-L. Cours au Collège de France, 2007-2010.

[42] Petrosjan, L. A. Cooperation in games with incomplete information. Nonlinear analysis and convex analysis, 469-479, Yokohama Publ., Yokohama, 2004.

[43] Simon, R. S., Spiez S. and Torunczyk, H. The existence of equilibria in certain games, for families of convex functions and a theorem of Borsuk-Ulam type, Israel Journal of Mathematics, 9, pp. 1-21, 1995.

[44] Sorin, S. A first course on zero-sum repeated games. Mathématiques \& Applications (Berlin), 37. Springer-Verlag, Berlin, 2002.

[45] Souquière A. Approximation and representation of the value for some differential games with imperfect information. To appear in Int. J. Game Theory, 2008.

[46] Souquière A. Jeux différentiels à information imparfaite. PhD Thesis, Brest University, 2010.

[47] Villani, C. Optimal transport : old and new, Springer, Berlin, 2009. 